\title{
Extraction of Plumes in Turbulent Thermal Convection
}

\author{
Emily S. C. Ching ${ }^{1}$, H. Guo ${ }^{1,2}$, Xiao-Dong Shang ${ }^{1,3}$, P. Tong ${ }^{3,4}$, and Ke-Qing Xia ${ }^{1}$ \\ ${ }^{1}$ Department of Physics, The Chinese University of Hong Kong, Shatin, Hong Kong \\ ${ }^{2}$ State Key Laboratory for Studies of Turbulence and Complex Systems, \\ Dept. of Mech. and Engin. Sci., Peking University, Beijing 100871, People's Republic of China \\ ${ }^{3}$ Department of Physics, Oklahoma State University, Stillwater, Oklahoma 74078 \\ ${ }^{4}$ Department of Physics, Hong Kong University of Science and Technology, Clear Water Bay, Kowloon, Hong Kong
}

(Dated: November 5, 2018)

\begin{abstract}
We present a scheme to extract information about plumes, a prominent coherent structure in turbulent thermal convection, from simultaneous local velocity and temperature measurements. Using this scheme, we study the temperature dependence of the plume velocity and understand the results using the equations of motion. We further obtain the average local heat flux in the vertical direction at the cell center. Our result shows that heat is not mainly transported through the central region but instead through the regions near the sidewalls of the convection cell.
\end{abstract}

PACS numbers: PACS numbers 47.27.-i

The Rayleigh-Bénard convection system consists of a closed cell of fluid heated from below and cooled on the top. The equations of motion, in Boussinesq approximation, are [1]:

$$
\begin{gathered}
\frac{\partial \mathbf{v}}{\partial t}+\mathbf{v} \cdot \nabla \mathbf{v}=-\nabla p+\nu \nabla^{2} \mathbf{v}+g \alpha \delta T \hat{\mathbf{z}} \\
\frac{\partial T}{\partial t}+\mathbf{v} \cdot \nabla T=\kappa \nabla^{2} T \\
\nabla \cdot \mathbf{v}=0
\end{gathered}
$$

where $\mathbf{v}$ is the velocity field, $p$ the pressure divided by density, $T$ the temperature field, and $\hat{\mathbf{z}}$ is the unit vector in the vertical direction. Furthermore, $\delta T=T-T_{0}$ where $T_{0}$ is the mean temperature of the bulk fluid, $g$ is the acceleration due to gravity and $\alpha, \nu$, and $\kappa$ are respectively the volume expansion coefficient, kinematic viscosity and thermal diffusivity of the fluid. The state of fluid motion is characterized by the geometry of the cell and two dimensionless parameters: the Rayleigh number, $\mathrm{Ra}=\alpha g \Delta L^{3} /(\nu \kappa)$, which measures how much the fluid is driven and the Prandtl number, $\operatorname{Pr}=\nu / \kappa$, which is the ratio of the diffusivities of momentum and heat of the fluid. Here $\Delta$ is the maintained temperature difference between the bottom and the top, and $L$ is the height of the cell. When Ra is sufficiently large, the convective motion becomes turbulent.

In turbulent convection, local velocity and temperature measurements taken at a point within the convection cell display complex fluctuations in time. On the other hand, visualization of the flow reveals recurring coherent structures. One prominent coherent structure is a plume, which is a mushroom-like flow generated by buoyancy. Thus at least two strategies can be employed to study turbulent thermal convection or turbulent flows in general. One is to analyze and understand the fluctuations of the local measurements. The other is to characterize the coherent structures and study and understand their dynamics. These two approaches are not independent but provide complementary knowledge of turbulent flows. In particular, there is the natural question of whether and how information about the coherent structures can be extracted from the local measurements.

For turbulent flows not driven by buoyancy, various methods including proper orthogonal decomposition, conditional sampling and wavelet analysis have been proposed to identify coherent vortical structures from local velocity measurements 2 . On the other hand, much less work has been done in identifying plumes or extracting information about plumes in turbulent thermal convection 3, 4, 5. Belmonte and Libchaber 3] used the skewness of the temperature derivative as a signature of the plumes. Zhou and Xia [4] associated the difference in the skewness of the positive and negative parts of the temperature difference with the presence of plumes and identified the plumes whenever the temperature difference becomes larger than a chosen threshold $[6]$. In Ref. [5], plumes are identified when each of the temperature, vertical velocity or vertical vorticity is larger than some threshold.

In this Letter, we present a scheme to extract information about plumes using simultaneous local velocity and temperature measurements. Our method makes explicit use of the physical intuition that plumes are generated by buoyancy and thus the velocity of a plume should correlate with the temperature fluctuation. Using this method, we obtain the temperature dependence of the plume velocity and the average local heat flux in the vertical direction at the cell center.

The experimental measurements that we use to illustrate and test our scheme of plume extraction were taken in an aspect-ratio-one cylindrical cell of height $L=$ $20.5 \mathrm{~cm}$ and filled with water 7]. The velocity $\mathbf{v}(t)$ was measured using a two-component laser Doppler velocimetry (LDV) system $[8]$ while the temperature $T(t)$ was measured using a small movable thermistor of $0.2 \mathrm{~mm}$ in diameter, $15 \mathrm{~ms}$ in time constant, and $1 \mathrm{mK} / \Omega$ in temperature sensitivity. The velocity and temperature measurements were simultaneously taken using a multi- 
channel LDV interface module to synchronize the data acquisition [7]. A triggering pulse from the LDV signal processor initiates the acquistion of an analog temperature signal. The spatial separation between the LDV focusing spot and the thermistor tip is kept at a minimal value of $0.7 \pm 0.2 \mathrm{~mm}$, a distance 3 times larger than the tip diameter of the thermistor but 20 times smaller than the correlation length between the temperature and velocity fluctuations [9]. The simultaneous velocity and temperature measurements can thus be assumed to be taken at the same location. In this work, we study measurements taken at two locations within the convection cell: the cell center and near the sidewall (on the midplane, at $8 \mathrm{~mm}$ from the sidewall). Near the sidewall, the vertical velocity component $v_{z}$ and one horizontal velocity component $v_{y}$, which is out of the rotation plane of the mean large-scale circulation, were measured. At the cell center, all three velocity components $v_{x}, v_{y}$ and $v_{z}$ were measured with $v_{x}$ along the direction of the large-scale mean circulation near the bottom plate. The number of velocity or temperature measurements at each location is $2-4 \times 10^{5}$, corresponding to 8 hours of real-time measurements.

From the simultaneous velocity and temperature measurements, we calculate $\langle\mathbf{v} \mid T(t)\rangle$, the conditional average of the velocity on the temperature measured at time $t$. In calculating $\langle\mathbf{v} \mid T(t)\rangle$, we take the average of those velocity measurements with the corresponding temperature measurements falling within $T(t) \pm 0.005^{\circ} \mathrm{C}$. Then we decompose the velocity measurement $\mathbf{v}(t)$ into a sum of $\langle\mathbf{v} \mid T(t)\rangle$ and the remaining part, denoted as $\mathbf{v}_{\mathbf{b}}(t)$ :

$$
\mathbf{v}(t)=\langle\mathbf{v} \mid T(t)\rangle+\mathbf{v}_{\mathbf{b}}(t)
$$

By construction, $\mathbf{v}_{\mathbf{b}}(t)$ is uncorrelated with $T(t)$ and averages to zero over time. Thus we take it as the background velocity fluctuation. If $\mathbf{v}(t)$ correlates with $T(t)$, $\langle\mathbf{v} \mid T(t)\rangle$ is different from the ordinary average velocity $\langle v\rangle$ and is a function of $T$ as well as $t$ since $T(t)$ depends on $t$. In this case, we define the velocity of the plume by $\mathbf{v}_{\mathbf{p}}(t) \equiv\langle\mathbf{v} \mid T(t)\rangle$. In other words, $\mathbf{v}_{\mathbf{p}}(t)$ is the part of $\mathbf{v}(t)$ that correlates with $T(t)$. If $\mathbf{v}(t)$ is uncorrelated with $T(t), \mathbf{v}_{\mathbf{b}}(t)=\mathbf{v}(t)-\langle\mathbf{v}\rangle$ and no plume is present. With this velocity decomposition, we can easily identify the local heat flux carried by the plumes. The normalized local heat flux is given by $\mathbf{j}(t)=[\mathbf{v}(t) \delta T(t)] L /(\kappa \Delta)$. The part carried by the plumes is thus $\mathbf{j}_{\mathbf{p}}(t) \equiv\left[\mathbf{v}_{\mathbf{p}}(t) \delta T(t)\right] L /(\kappa \Delta)$. When the contribution by the plumes is subtracted, the remaining is the heat flux carried by the background velocity fluctuation, which is $\mathbf{j}_{\mathbf{b}}(t)=\left[\mathbf{v}_{\mathbf{b}}(t) \delta T(t)\right] L /(\kappa \Delta)$ and averages to zero over time.

It is found that the horizontal velocity components have little correlation with the temperature fluctuation, having a correlation coefficient of 0.1 and 0.2 respectively at the cell center and near the sidewall. On the other hand, the vertical velocity component $v_{z}$ shows stronger correlation with $T(t)$, having a correlation coefficient of 0.3 and 0.5 respectively at the cell center and near the sidewall. Consistently, we find that the difference between $\langle\mathbf{v} \mid T(t)\rangle$ and $\langle\mathbf{v}\rangle$ lies mainly in the vertical direction. That is, $\mathbf{v}_{\mathbf{p}}(t) \approx v_{p z}(t) \hat{\mathbf{z}}$.

It was recently found [7] that the probability distribution of the local heat flux in the horizontal direction is approximately symmetric whereas that in the vertical direction is skewed towards the positive fluctuation. This asymmetry in the vertical flux distribution was interpreted to be caused by the plumes [7]. We use these results to test our scheme of plume extraction. We expect that after subtracting the the contribution by the plumes, the probability distributions of the local heat flux in the horizontal and vertical directions would become identical. In Fig. 1 we show the probability distributions of $\tilde{j}_{b x}, \tilde{j}_{b y}$, and $\tilde{j}_{b z}$. Here the standardized variable $\tilde{X}$ is defined as $(X-\langle X\rangle) / \sigma_{X}$ where $\langle X\rangle$ and $\sigma_{X}$ are respectively the mean and standard deviation of $X$. In the calculation, we estimate the mean temperature of the bulk fluid $T_{0}$ by the average temperature measured at the cell center. As expected, the standardized distributions of the horizontal and vertical components of $\tilde{\mathbf{j}_{\mathrm{b}}}$ are the same. Moreover, they are approximately the same at the two locations studied.

In a recent study [10], the three velocity components at the cell center were found to possess the She-Leveque hierarchical structure 11]:

$$
\frac{S_{p+2}(\tau)}{S_{p+1}(\tau)}=A_{p}\left[\frac{S_{p+1}(\tau)}{S_{p}(\tau)}\right]^{\beta}\left[S^{(\infty)}(\tau)\right]^{1-\beta}
$$

where $S_{p}(\tau) \equiv\left\langle|v(t+\tau)-v(t)|^{p}\right\rangle$ with $v$ being one of the three velocity components, $A_{p}$ are constants independent of $\tau$ and $S^{(\infty)}(\tau) \equiv \lim _{p \rightarrow \infty} S_{p+1}(\tau) / S_{p}(\tau)$. Here, $\beta$ is a parameter whose value lies between 0 and 1 . The smaller $\beta$ is, the more intermittent the velocity fluctuation is. It was found that the two horizontal velocity components are characterized by the same value of $\beta$ but the vertical velocity component is characterized by a smaller value of $\beta$. This distinction was attributed 10 to the presence of the plumes which makes the vertical velocity component more intermittent. As a further test of our scheme of plume extraction, we analyze $\mathbf{v}_{\mathbf{b}}(t)$ at the cell center to check whether its horizontal and vertical components will now be characterized by the same value of $\beta$. To get the value of $\beta$, we follow the procedure developed in Ref. 12] and calculate the relative scaling exponents $\rho(p)$ of the normalized structure functions, defined by

$$
\frac{S_{p}(\tau)}{S_{2}(\tau)^{p / 2}} \sim\left[\frac{S_{1}(\tau)}{S_{2}(\tau)^{1 / 2}}\right]^{\rho(p)}
$$

If Eq. (5) holds, we have 12 $\Delta \rho(p+1)=\beta \Delta \rho(p)-$ $(1+\beta)$, where $\Delta \rho(p) \equiv \rho(p+1)-\rho(p)$. In Fig. 2] we plot $\Delta \rho(p+1)$ vs $\Delta \rho(p)$. It can be seen that the data points for the three velocity components of $\mathbf{v}_{\mathbf{b}}(t)$ nearly 

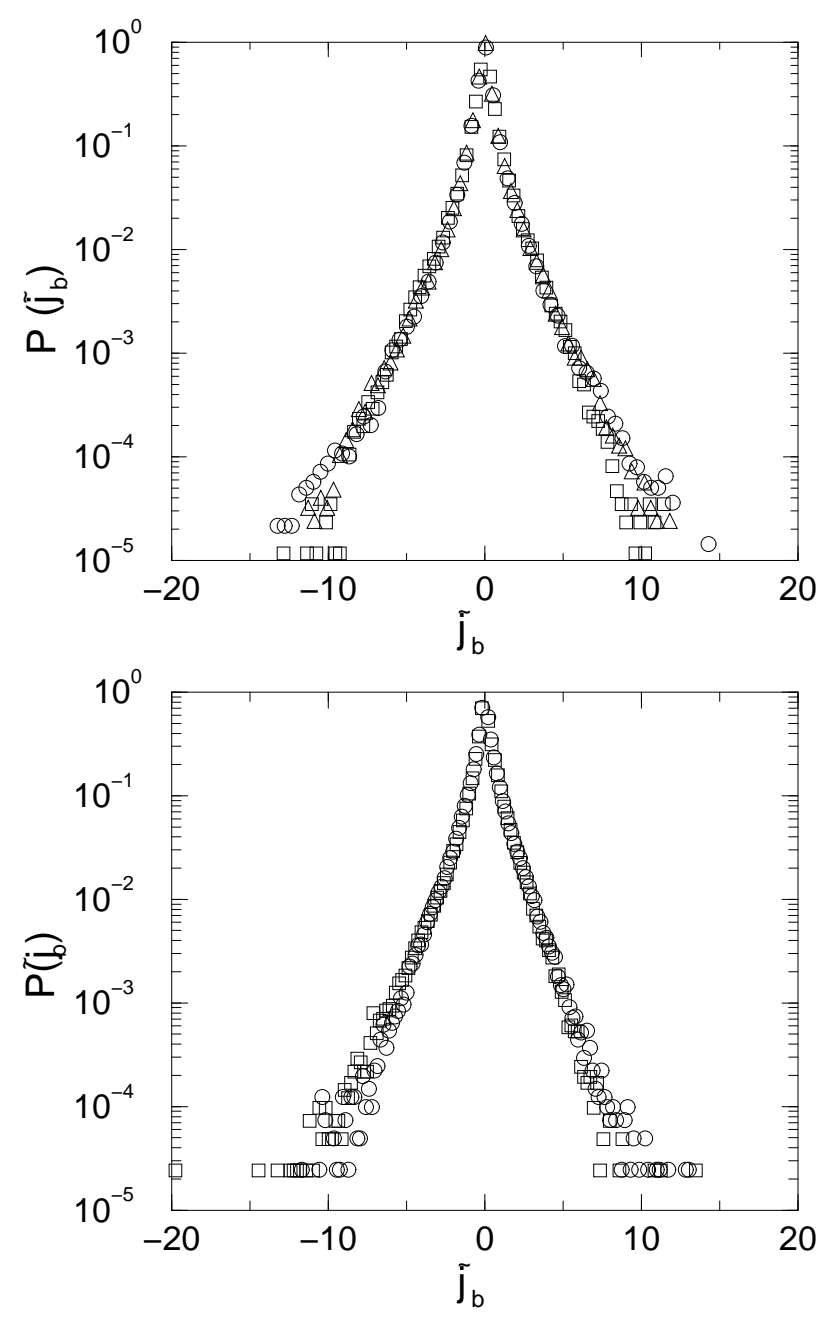

FIG. 1: The standardized probability density functions $P\left(\tilde{j}_{b x}\right)$ (triangles), $P\left(\tilde{j}_{b y}\right)$ (squares), and $P\left(\tilde{j}_{b z}\right)$ (circles) at $\mathrm{Ra}=2.6 \times 10^{9}$. The measurements were made at the cell center (top panel) and near the sidewall (bottom panel).

fall on the same line, showing that the difference in the $\beta$ values of the horizontal and vertical components indeed becomes vanishingly small.

Next we use our scheme of plume extraction to study the temperature dependence of the plume velocity. The results are shown in Fig. 3. We find that $v_{p z}=a \delta T$ at the cell center and $v_{p z}=b \sqrt{T-T_{s}}$ for $T>T_{0}\left(>T_{s}\right)$ near the sidewall for some constants $a$ and $b$. The fitted value of $T_{s}$ differs from the value of $T_{0}$ as estimated by the average temperature at the cell center by about $1 \%$. We believe that this difference is caused by drifts in the mean temperature of the bulk fluid over time and that the measurements at the two locations were not taken at the same time. Hence, it is reasonable to conclude that $v_{p z} \propto \sqrt{\delta T}$ for $\delta T>0$ near the sidewall.

In the following, we shall understand this temperature dependence. At the cell center, the mean velocity vanishes and thus we expect the viscous term to dominate

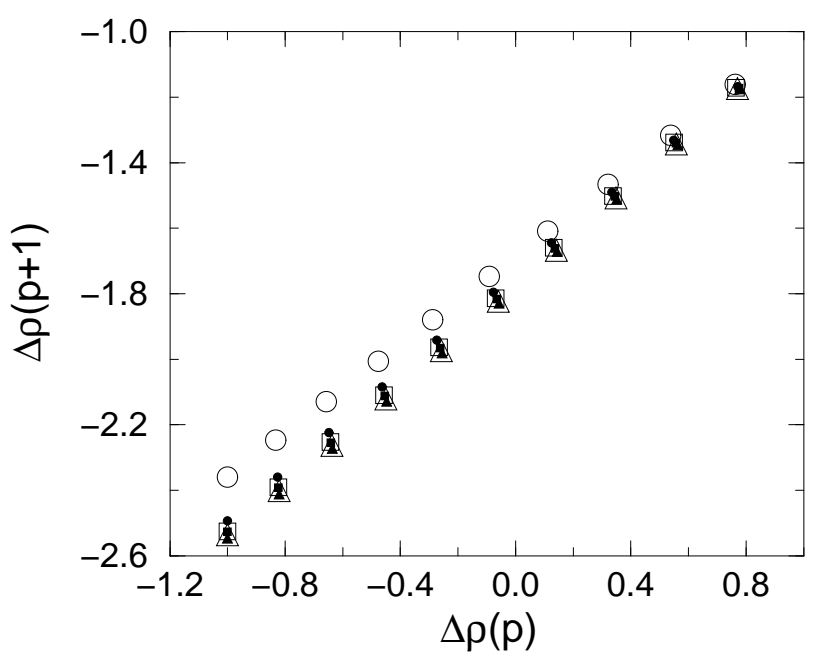

FIG. 2: $\Delta \rho(p+1)$ vs $\Delta \rho(p)$ for the $x$-component (triangles), $y$ component (squares), and $z$-component (circles) for $\mathbf{v}$ (open symbols) and $\mathbf{v}_{\mathbf{b}}$ (filled symbols) at the cell center for $\mathrm{Ra}$ $=4.8 \times 10^{9}$.

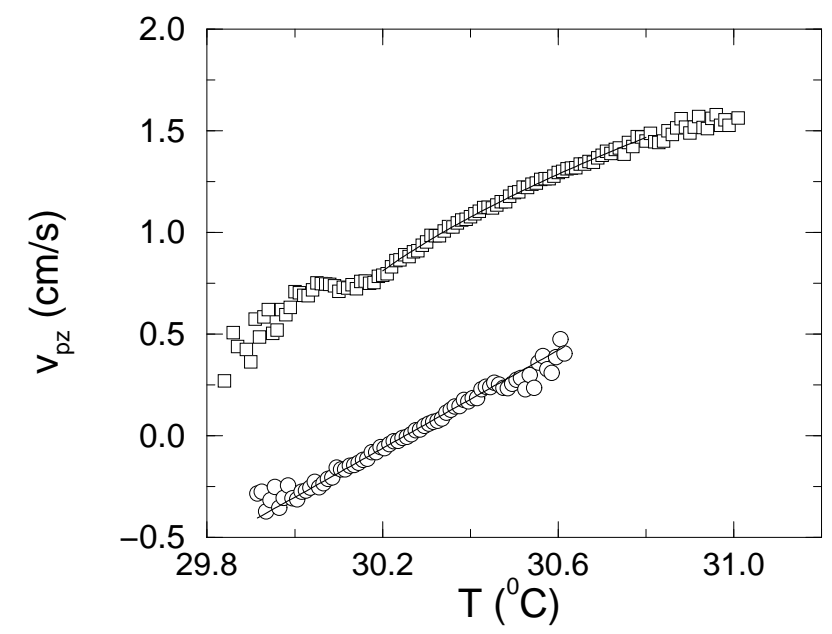

FIG. 3: $v_{p z}$ vs $T$ at the cell center (circles) and near the sidewall (squares) at $\mathrm{Ra}=2.6 \times 10^{9}$. The solid lines from bottom to top are respectively the fits of $a \delta T$ and $b \sqrt{T-T_{s}}$ for some constants $a$ and $b$. The fitted value for $T_{s}$ is $29.9^{\circ} C$ while $T_{0}$ is $30.2^{\circ} \mathrm{C}$.

over the advection term. By balancing the viscous term by the buoyancy term in Eq. (1), we have

$$
\text { center : } \quad \frac{\nu v_{p z}}{l_{c}^{2}}=g \alpha \delta T
$$

for some length scale $l_{c}$, which explains the temperature dependence observed at the cell center. Since plumes are excitations from the thermal boundary layer, we expect $l_{c}$ to be of the order of the thermal boundary layer thickness $\lambda_{t h}$. We calculate $l_{c}$ from the fitted value of $a$ and compare $l_{c} / L$ with $\lambda_{t h} / L$ 13. As shown in Fig. 团 $l_{c} / L$, after scaled down by a factor of about 2 , coincides with 


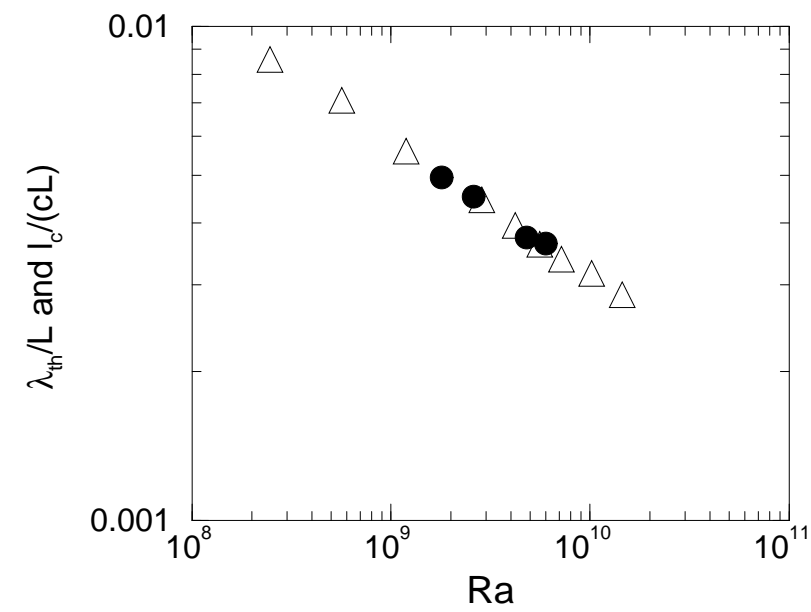

FIG. 4: $\lambda_{t h} / L$ (triangles) [13] and $l_{c} /(c L)$ (filled circles) vs Ra with $c=1.9$.

$\lambda_{t h} / L$ over the limited Ra range studied.

Near the sidewall, the average velocity of the plumes is equal to the average vertical velocity, which is essentially the mean large-scale circulation velocity and is relatively large. Thus the advection term should dominate over the viscous term. For $\delta T>0$, we equate the advection term by the buoyancy term in Eq. (11) and get

$$
\text { sidewall : } \quad \frac{v_{p z}^{2}}{l_{s}}=g \alpha \delta T \quad \text { for } \delta T>0
$$

for some other length scale $l_{s}$. Hence $v_{p z} \propto \sqrt{\delta T}$ for $\delta T>$ 0 reproducing the temperature dependence observed near the sidewall. Since the advection term is produced by the mean large-scale circulation, it seems reasonable to expect $l_{s}$ to be of the order of the cell height $L$. Indeed $l_{s}$, calculated from the fitted value of $b$, is about $0.3-0.4 L$ and has little dependence on Ra.

We further obtain the average local heat flux in the vertical direction at the cell center, $\left\langle j_{z}(t)\right\rangle_{c}$, which can be thought of as a pointwise Nusselt number at the cell center. Since $\left\langle\mathbf{j}_{\mathbf{b}}(t)\right\rangle=0,\left\langle j_{z}(t)\right\rangle=\left\langle j_{p z}(t)\right\rangle=$ $\left\langle v_{p z}(t) \delta T(t)\right\rangle L /(\kappa \Delta)$. Using (7), we have

$$
\left\langle j_{z}(t)\right\rangle_{c}=\operatorname{Ra} \frac{\left\langle(\delta T)^{2}\right\rangle_{c}}{\Delta^{2}}\left(\frac{l_{c}}{L}\right)^{2}
$$

Note that $\Delta_{c} \equiv \sqrt{\left\langle(\delta T)^{2}\right\rangle_{c}}$ is the root-mean-square temperature fluctuation at the cell center. It was found that $\Delta_{c} / \Delta \sim \mathrm{Ra}^{-1 / 7}$ both in low temperature helium gas 14] and in water 15]. As $l_{c} / L$ goes like $\lambda_{t h} / L$ (see Fig. (4) and $\lambda_{t h} / L \sim \mathrm{Ra}^{-2 / 7}\left[13\right.$, we have $\left\langle j_{z}(t)\right\rangle_{c} \sim$ $\mathrm{Ra}^{1 / 7}$. The scaling exponent $1 / 7$ is clearly different from the scaling exponent of approximately $2 / 7$ of the Nusselt number 16. This result thus shows explicitly that heat cannot be mainly transported through the central region of the convection cell.
In summary, we have presented a scheme to extract information about plumes, the prominent coherent structures in turbulent thermal convection. Our scheme involves a decomposition of the local velocity into two parts. The part that correlates with the local temperature measured at the same time is defined as the plume velocity. Using this scheme of plume extraction, we have found the temperature dependence of the plume velocity at the center and near the sidewall of the convection cell, and understood such dependence from the equations of motion. Moreover, we have obtained the average local heat flux in the vertical direction at the cell center, and found that it has a scaling dependence with Ra different from that of the Nusselt number. This difference shows that heat is not mainly transported through the central region but instead through regions near the sidewalls of the convection cell. Further results about the mean largescale circulation velocity and the velocity fluctuation at the cell center can be derived from Eqs. (7) and (8), and will be reported elsewhere.

The work at the Chinese University of Hong Kong was supported by the Hong Kong Research Grants Council (ESCC and HG by CUHK 4046/02P and XDS and KQX by CUHK $4242 / 01 \mathrm{P})$. PT was supported in part by NSF0071823 and HKUST 603003.

[1] See, for example, Landau and Lifshitz, Fluid Mechanics (Pergamon Press, Oxford, 1987).

[2] See, for example, J.P. Bonnet et. al., Exp. Fluids 25, 197 (1998).

[3] A. Belmonte and A. Libchaber, Phys. Rev. E 53, 4893 (1996).

[4] S.-Q. Zhou and K.-Q. Xia, Phys. Rev. Lett. 89, 184502 (2002).

[5] K. Juliem, S. Legg, J. McWilliams, and J. Werne, J. Fluid Mech. 391, 151 (1999).

[6] F. Moisy, H. Willaime, J.S. Andersen, and P. Tabeling, Phys. Rev. Lett. 86, 4827 (2001).

[7] X.-D. Shang, X.-L. Qiu, P. Tong, and K.-Q. Xia, Phys. Rev. Lett. 90, 074501 (2003).

[8] X.-L. Qiu and P. Tong, Phys. Rev. E 64, 036304 (2001); 66, 026308 (2002).

[9] The correlation length was obtained by varying the separation between the temperature and velocity probes.

[10] E.S.C. Ching, C.K. Leung, X.-L. Qiu, and P. Tong, Phys. Rev. E 68, 026307 (2003).

[11] Z.-S. She and E. Leveque, Phys. Rev. Lett. 72, 336 (1994).

[12] E.S.C. Ching, Z.-S. She, W. Su, and Z. Zou, Phys. Rev. E 65066303 (2002).

[13] S.-L. Lui and K.-Q. Xia, Phys. Rev. E 57, 5494 (1998).

[14] B. Castaing et al., J. Fluid Mech. 204, 1 (1989).

[15] K.-Q. Xia and S.-L. Lui, Phys. Rev. Lett. 79, 5006 (1997).

[16] See, for example, S. Grossmann and D. Lohse, J. Fluid Mech. 407, 27 (2000) for a review. 